\title{
Innovación en Telemedicina: una oportunidad alternativa para el aumento de la cobertura en salud
}

\author{
Telemedicine Innovation: An alternative Opportunity to Increase \\ Health Coverage
}

\section{Inovação em Telemedicina: uma oportunidade alternativa para o aumento no atendimento da saúde}

La creación y masificación de nuevas tecnologías inevitablemente genera cambios significativos en el mundo. La Cibermedicina es la aplicación de redes de cubrimiento global a la Medicina y la Salud Pública, encargada de evaluar las oportunidades e implicaciones de la internet y demás tecnologías asociadas (1). La Salud Web 2.0 representa una modalidad sanitaria innovadora donde los usuarios que interactúan en la red de internet asumen un rol más activo, desde la resolución de inquietudes hasta la satisfacción de necesidades que promueven estilos de vida saludables, reflejándose en estudios que demuestran un mejoramiento en las actitudes, conocimientos y conductas en salud relacionados con nutrición y consumo de tabaco principalmente (2).

La Organización Mundial de la Salud define a la Telemedicina como la prestación de servicios de salud a distancia por profesionales que hacen uso de las Tecnologías de la Información y la Comunicación (TIC), a través de la prevención, diagnóstico y tratamiento de enfermedades; se incluyen la investigación y la educación con el fin de conseguir avances en la salud de las comunidades y los individuos (3). La Organización Panamericana de la Salud incluyó los términos en alusión a la Telesalud en 1997 y en el año 2005 estableció el fortalecimiento de alianzas públicas y privadas dentro de un marco jurídico para garantizar la ética, seguridad y calidad de la misma (4). Una red de Telemedicina está compuesta por recurso humano profesional, centros de salud de referencia con oferta de diferentes especialidades y equipos que cuenten con tecnología en medios de transmisión (5).
Desde hace más de tres décadas se habla de Telemedicina alrededor del mundo, con el principal objetivo de fomentar la equidad en el acceso a los servicios de salud. El abordaje de pacientes a través de internet con entrevista e historia clínica en línea para orientar el diagnóstico y manejo de pacientes se ha estado practicando en países como Holanda, Suecia, Estados Unidos, Canadá, Inglaterra, Italia, España y países escandinavos. En Australia se ha destacado un enfoque particular en instituciones de Telemedicina hacia el manejo de pacientes quemados. En Grecia, Portugal e Israel se destaca la Telecardiología infantil. Francia y Alemania han mostrado amplia experiencia en Telecirugía robótica. La Unión Internacional de Telecomunicaciones opera en una red de hospitales entre África y centros europeos junto con el Grupo de Colaboración Europea, pero desafortunadamente, las zonas que pueden beneficiarse muchas veces no cuentan con los recursos para llevar a cabo la aplicación de estas tecnologías en salud. (6).

Existe una red de Telemedicina que opera directamente entre una empresa de Suiza y países latinoamericanos con el fin de ofrecer servicios de Telecardiología (holter de presión arterial y electrocardiografía) y pruebas transtelefónicas respiratorias funcionales (4). En México, más de 15 entidades prestan servicios de teleeducación y telediagnóstico en aproximadamente 150 unidades desde la última década, con más de 10 especialidades médicas ofrecidas en teleasistencia y de manera remota con sistemas de telecomunicaciones, desarrollando jornadas de electrocardiografía, electroencefalografía, ultrasonido y colposcopias. En Venezuela se ha procurado mantener 
conexión a internet en zonas rurales y remotas a través del satélite VeneSat para prestar servicios de Telemedicina y capacitación al personal que reside en regiones aisladas. En Ecuador se realizan servicios de telemonitoreo y teleconsultas por medio de la red de Cooperación Latinoamericana de Redes Avanzadas (CLARA) (1).

En Colombia hay grandes extensiones de zonas aisladas donde, por ubicación geográfica, falta de uniformidad en la distribución de recurso humano profesional o áreas de conflicto armado la población se encuentra sin programas de cobertura en salud, tanto en el sector público como en el privado, por lo cual la Telemedicina es probablemente el único sistema alternativo para contrarrestar la falta de accesibilidad (4,7). Con el Centro de Telemedicina de Colombia se han desarrollado programas dirigidos a la teleasistencia de víctimas de minas antipersonas, no solo para guiar acciones en casos de emergencia sino también para su respectiva rehabilitación, junto con la red del Centro de Rehabilitación Internacional (6). Se han adoptado plataformas por medio de las cuales se ofrece teleeducación en salud con herramientas de Medicina 2.0 en más de 30 instituciones, incluyendo teleconsultas y telequirófano con entidades remisoras extranjeras; cirujanos que no residen en el mismo país o incluso que viven en otro continente pueden conducir robots con telemandos a kilómetros de distancia. Se trabaja de la mano con el Enlace Hispano Americano de Salud (EHAS), una red con la que se pretende cubrir las necesidades de los diferentes países, desde aquellos que en determinado momento puedan proveer los diferentes servicios $(1,8)$.

Dentro de las ventajas que ofrece la Telemedicina se identifican la reducción de costos para el sistema de salud y los usuarios que deben transportarse regularmente a lugares distantes para asistir a citas de control; se provee un enfoque más preventivo, diagnósticos oportunos, seguimiento más constante a pacientes con enfermedades crónicas y de difícil adherencia al manejo y mayor acompañamiento al personal que presta servicios de salud a grupos sociales marginados $(7,9)$.

Como desventajas, se altera de manera considerable la relación médico-paciente aunque se contrarresta la dificultad y demora en la prestación convencional de servicios de salud; se presenta un riesgo potencial respecto a la seguridad y los límites de acceso a la información de los pacientes, la evasión en la implementación de protocolos de atención y la falta de políticas para acceso a la información. Por estas razones, el ejercicio de la Telemedicina debe convertirse en responsabilidad gubernamental, de centros académicos y de organizaciones públicas y privadas, con medidas de seguridad que protejan a los pacientes y usuarios en general, garantizando así la calidad en la atención, integridad y confidencialidad de la información, brindando seguridad, equidad e implementando cambios constantes para el mejoramiento $(3,4,5,9,10)$.
Dentro de los principales estándares regulatorios a nivel internacional se destacan: La Ley Health Insurance Portability and Accountability (HIPPA), los Objetivos de Control para la Información y Tecnologías (COBIT), el Informe de Revisión de Información e Identificación del Paciente (CALDICOT), la Organización Internacional de Telecomunicaciones (ITU-T) y las normas ISO 27000. El Ministerio Nacional de Protección social emitió la Resolución 1448 en el año 2006, con la cual se busca regular, establecer las condiciones de cumplimiento obligatorio y garantizar la calidad en instituciones que presten servicios de salud en Telemedicina, aludiendo a ella como una modalidad de servicio (5).

La educación sanitaria resulta fundamental para enfrentar la crisis que actualmente atraviesan los sistemas sanitarios. Las herramientas Web 2.0 y los dispositivos Personal Digital Assistant (PDA) se presentan como instrumentos facilitadores de información y conocimiento en salud tanto para profesionales como para estudiantes y pacientes en general. Un estudio que incluyó más de 85,000 consultas, de las cuales el $60 \%$ se llevó a cabo con Telemedicina, reportó dentro de las especialidades más consultadas: Salud Mental, Cardiología, Dermatología, Pediatría y Ortopedia. Estas fuentes de generación de teorías deben ser corroboradas por entidades acreditadas que exijan rigurosidad científica, pues dicha información no puede considerarse válida para la toma de decisiones sin antes ser revisada. Cabe resaltar el valor educativo de proyectos que pretenden enriquecer la formación en salud con base en simuladores de realidad virtual y neuroarquitectura que generen ambientes inmersivos para el desarrollo de habilidades profesionales y que puedan implementarse con programas de teleeducación $(1,6,11)$.

Es clara la importancia de formar una sólida coalición entre los principales entes que deben involucrarse para que los profesionales de la salud adapten sus aptitudes en pro de una mayor autonomía del paciente a través de la telesalud, no solo para beneficio de las poblaciones más apartadas, sino también de la sociedad en general $(12,13,14)$. Se deben implementar metodologías con un proceso continuo de educación y socialización del personal de salud técnico y clínico, basándose en evidencias e impulsando la toma de decisiones adecuadas y oportunas, integrando los resultados obtenidos y un vasto marco conceptual con protocolos que involucren el mejoramiento y la innovación de tendencias tecnológicas en salud, lo cual se traduce en un potencial crecimiento en la calidad de vida de las personas y, por ende, poblaciones más saludables $(8,15)$.

$M^{\text {a }}$ Catalina De Montijo V., MD. Grupo de Investigaciones Clínicas UNAB Grupo de Investigación CARING UNAB Universidad Autónoma de Bucaramanga Correo electrónico: mde4@unab.edu.co 


\section{Referencias}

1. Viloria-Núñez C. Tecnologías de la información para la educación, investigación y aplicación en el área de la salud. Bondades y retos. Salud 2009; 25(2): 331-349.

2. Fernández-Silano M. La Salud 2.0 y la atención de la salud en la era digital. Revista médica Risaralda. 2014; 20(1): 41-46.

3. Santamaría-Puerto G, Hernández-Rincón E. Aplicaciones Médicas Móviles: definiciones, beneficios y riesgos. Salud 2015; 31(3): 599-607.

4. Matiz-Camacho $\mathrm{H}$. El futuro de la medicina con equidad en Colombia: La Telemedicina. Rev Col Cardiol 2007; 14(1): 1-8.

5. Guillen-Pinto EP; Ramírez López LJ, Estupiñán EP. Análisis de seguridad para el manejo de la información médica en Telemedicina. Cienc Ing Neogranad 2011; 21(2): 57-89.

6. Agámez-Luengas S, Aldana-Bolaño M, Barreto-Arco V, Santana-Goenaga A, Caballero-Uribe CV. Aplicación de nuevas tecnologías de la información en la enseñanza de la medicina. Salud Uninorte 2009; 25(1): 150-171.

7. De Ortúzar MG. Igualdad de acceso a la telesanidad en zonas rurales y aisladas: propuesta de un marco ético normativo integral de acceso y distribución. Rev latinoam bioet 2009; 9(1):76-93.

8. Carvajal-Tejada M, Ruiz-lbañez CG. Evaluación técnica y clínica de tecnología biomédica en procesos de adquisición: un enfoque en evaluación de tecnologías en salud. Rev ing biomed 2008; 2(4): 34-45.

9. Kvedar J, Coye MJ, Everett W. Connected health: a review of technologies and strategies to improve patient care with telemedicine and telehealth. Health Aff (Millwood) 2014; 33(2):194-199. Doi: 10.1377/hlthaff. 2013.0992

10. Schwamm LH. Telehealth: seven strategies to successfully implement disruptive technology and transform health care. Health Aff (Millwood) 2014; 33(2):200-206. Doi: 10.1377/hlthaff.2013.1021

11. Hege I, Kononowicz AA, Tolks D, Edelbring S, Kuehlmeyer K. A qualitative analysis of virtual patient descriptions in healthcare education based on a systematic literature review. BMC Med Educ 2016; 16:146. Doi: 10.1186/s12909-016-0655-8

12. MacNeill V, Sanders C, Fitzpatrick R, Hendy J, Barlow J, Knapp M, Rogers A, Bardsley M, Newman SP. Experiences of front-line health professionals in the delivery of telehealth: a qualitative study. $\mathrm{Br} \mathrm{J}$ Gen Pract 2014;64(624):e401-7. Doi: 10.3399/bjgp14X680485.

13. Victorson D, Banas J, Smith J, Languido L, Shen E, Gutierrez S, Cordero E, Flores L. eSalud: designing and implementing culturally competent ehealth research with latino patient populations. Am J Public Health 2014; 104(12):2259-2265. Doi: 10.2105/AJPH.2014.302187

14. Moorhead SA, Hazlett DE, Harrison L, Carroll JK, Irwin A, Hoving C. A new dimension of health care: systematic review of the uses, benefits, and limitations of social media for health communication. J Med Internet Res 2013;15(4): e85. Doi: 10.2196/jmir.1933

15. Agarwal S, Perry HB, Long LA, Labrique AB. Evidence on feasibility and effective use of mHealth strategies by frontline health workers in developing countries: systematic review. Trop Med Int Health 2015; 20(8):1003-14. Doi: 10.1111/tmi.12525 